Journal of Social Sciences 7 (4): 565-568, 2011

ISSN 1549-3652

(C) 2011 Science Publications

\title{
An Analysis of Performance in Elite Young Cross-Country Skiing: Classical Style Vs Skate Skiing
}

\author{
${ }^{1}$ Francisco Pradas de la Fuente, ${ }^{1}$ Carlos Castellar Otin, \\ ${ }^{1}$ Luis Manzano Benito, ${ }^{1}$ Jose Manuel Lou Bonafonte, \\ ${ }^{1}$ Marta Rapun Lopez, ${ }^{2}$ Luis Poch Galloso and ${ }^{3}$ Balint Gheorghe \\ ${ }^{1}$ Faculty of Sciences of Health and Sports, University of Zaragoza, Spain \\ ${ }^{2}$ Technical Center in Winter Sports of Aragon, Spain. \\ ${ }^{3}$ Faculty of Health, Sports and Human Movement Sciences, \\ University "Vasile Alecsandri”of Bacau, Romania
}

\begin{abstract}
Problem statement: The aim of this study was to assess the differences in the skiing performance of two skiing styles (classical style Vs. skate skiing), in a group of young high-level skiers. Approach: Four high-level male junior cross-country skiers from the Centre of Winter Sports of Aragon (Spain), were selected for this study that was developed during two competitions: $10 \mathrm{~km}$ free technique and $10 \mathrm{~km}$ classical technique performed in the same circuit and under the same conditions (snow quality, temperature, wind and relative humidity). Physiological variables, maximal and medium heart rate, blood lactate concentration (basal, final and during the recovery, after 2, 4, 6, 8 and $10 \mathrm{~min}$ ) and medium skiing speed, were analyzed with GPS Garmin Forerruner $305^{\circledR}$ Heart Rate Pulse and Lactate Dr. Lange analyzer ${ }^{\circledR}$. Normally distributed data (Kolmogorov-Smirnov Test and Shapiro-Wilk) were statistically analyzed with one way ANOVA and t-Test for post-hoc comparisons $(\mathrm{p}<0.05)$. No-normally distributed data were analyzed with the Friedman and Wilcoxon Tests $(\mathrm{p}<0.05)$. Results: No significant differences have been recorded in the medium skiing speed, maximal and medium heart rate between the two styles. The peak heart rate was the same in both styles (188 bpm), although medium heart rate was lower in skate skiing vs. classical skiing (165 bpm Vs. $175 \mathrm{bpm}$ ). Blood lactate concentrations recorded significant differences during 4, 6,8 and 10 min of recovery, being higher in free technique $\left(12.7 \pm 1.7 ; 11.4 \pm 1.8 ; 10.4 \pm 1.4\right.$ and $8.9 \pm 1.6 \mathrm{mmol} \mathrm{L}^{-1}$ Vs. $8.7 \pm 1.3$; $7.5 \pm 1.6 ; 6.3 \pm 1.3$ and $5.6 \pm 1.1 \mathrm{mmol} \mathrm{L}^{-1}$, respectively). Conclusion: The results of this study suggest that skate skiing generates a higher impact on the cross-country skier than classical skiing, due to higher blood lactate concentrations during 4, 6, 8 and $10 \mathrm{~min}$ of recovery. The own characteristics of the free technique may be the reason of the lower mean heart rate.
\end{abstract}

Key words: Cross-country skiing, classical style, skate skiing, lactate, heart rate

\section{INTRODUCTION}

Cross-country skiing is characterized by two different styles: the classical style and the skate skiing. The classical technique consists of alternating arm and legs in a rhythmic way and involves movements of the skis in a parallel direction. Ski skating incorporates a lateral pushing action with one ski while the opposite one maintains a forward glide (Vaananen, 2004). Both styles present maximal, submaximal and continuous efforts of changing intensity as a consequence of the particular orography of the race circuits with flat, up and down hills. These changes also produce variability in the predominant metabolism. Metabolism depends on length competition, although the cross-country skiers maintain efforts greater than anaerobic threshold, keeping the physical activity near to the maximal oxygen consumption. However, the two techniques require different physical and physiological demands on the skier.

The aim of this study was to assess the differences in the performance of cross-country skiing comparing classical versus skate skiing styles in a group of elite young cross-country skiers.

\section{MATERIALS AND METHODS}

Four high-level male junior cross-country skiers belonging to the Technical Center of Winter Sports (CETDI, Jaca, Spain) were selected for the study. The

Corresponding Author: Francisco Pradas de la Fuente, Faculty of Sciences of Health and Sports, University of Zaragoza, Spain 
average age was $17.7 \pm 1.4$ years, with a weight of 64.3 $\pm 6.1 \mathrm{kgs}$ and an average height of $175.5 \pm 0.05 \mathrm{cms}$. The study was performed in the Linza cross-country skiing circuit (Huesca, Spain), during Aragon Crosscountry Championship.

During the competition the heart rate was analysed every five seconds, recording the maximal heart rate and the average heart rate. Heart rate was also recorded during recovery after the race, with a watch that includes Global Position System (GPS) and heart rate pulse (Garmin Forerunner ${ }^{\circledR}$ 305, USA). This method allows measurements in different orography and complexity will be greatly reduced (Azar and Shafri, 2009). Lactate levels where also analysed by using an absorption method (Dr Lange ${ }^{\circledR}$ Photometer LP 450, Berlin, Germany) three hours before the race (basal), immediately after the finishing the skier and after 2, 4, 6,8 and $10 \mathrm{~min}$ of recovery. A $10 \mu \mathrm{L}$ capillary blood sample was taken from at the ear lobe for a measurement of blood lactate concentration.

After assessing normal data distribution (Kolmogorov-Smirnov and Shapiro-Wilk tests), data were statistically analyzed with One Way ANOVA and $\mathrm{t}$-Test for post-hoc comparisons $(\mathrm{p}<0.05)$. No-normally distributed data were analyzed with the Friedman Test and Wilcoxon Test $(\mathrm{p}<0.05)$. The data were analysed with the Statistical Package for Social Science (SPSS ${ }^{\circledR}$, Chicago, IL, USA). Results in the text and tables are given as the mean standard \pm deviation.

\section{RESULTS}

Table 1 summarized the results of the competitive cross-country skiing races (10-km for both techniques). The time recorded for completing the races was of 28:30 min in the skate skiing and 29:29 $\mathrm{min}$ in the classical style. Significant differences between the two styles were found in the four different measurements of recovery blood lactate.

Figure 1 shows the average speed of the styles (skate Vs. classical). No significant differences were found between the two styles $\left(20.64 \pm 0.3 \mathrm{~km} \mathrm{~h}^{-1} \mathrm{Vs}\right.$ $20.09 \pm 0.9 \mathrm{~km} \mathrm{~h}^{-1}$ respectively).

Maximal blood lactate levels are presented in Figure 2. Lactate concentration was higher in skating style vs. classic style $\left(13.7 \pm 2 \mathrm{mmol} \mathrm{L}^{-1}\right.$ Vs. $11.9 \pm 0.8$ mmol $\left.\mathrm{L}^{-1}\right)$, although it was not statistically different.

Lactate levels measured during recovery are depicted in Figure 3. Significant differences between the skate and the classicl skiing style were recorded after $4 \mathrm{~min}\left(12.7 \pm 1.7 \mathrm{mmol} \mathrm{L}^{-1} \mathrm{Vs} .8 .7 \pm 1.3 \mathrm{mmol}\right.$ $\left.\mathrm{L}^{-1}\right), 6 \mathrm{~min}\left(11.4 \pm 1.8 \mathrm{mmol} \mathrm{L}{ }^{-1}\right.$ Vs. $7.5 \pm 1.6 \mathrm{mmol}$
$\left.\mathrm{L}^{-1}\right), 8 \mathrm{~min}\left(10.4 \pm 1.4 \mathrm{mmol} \mathrm{L}{ }^{-1} \mathrm{Vs} .6 .3 \pm 1.3 \mathrm{mmol}\right.$ $\left.\mathrm{L}^{-1}\right)$ and $10 \mathrm{~min}\left(8.9 \pm 1.6 \mathrm{mmol} \mathrm{L}{ }^{-1} \mathrm{Vs} .5 .6 \pm 1.1\right.$ $\left.\mathrm{mmol} \mathrm{L}{ }^{-1}\right)$ of recovery, respectively.

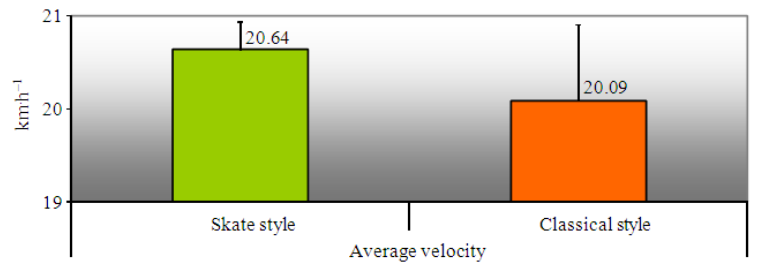

Fig. 1: Comparative to the average speed between the skate style and the classic style

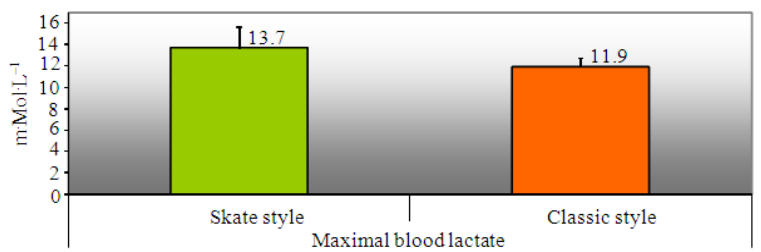

Fig. 2: Comparative to the lactate levels between the skate style and the classic style

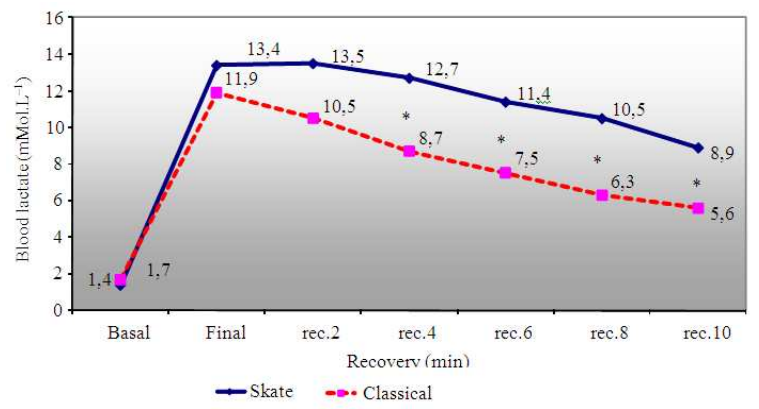

Fig. 3: Comparison of blood lactate curve in skate style and classical before, final and during the event recovery (min. 2, 4, 6, 8 and 10). * Statistical differences $(\mathrm{p}<0.05)$

Table 1: Results of race performance (Skate: skate style, Classic: classic style)

\begin{tabular}{|c|c|c|}
\hline Parameter & Skate & Classical \\
\hline Average speed $\left(\mathrm{km} \mathrm{h}^{-1}\right)$ & $20.64 \pm 0.3$ & $20.09 \pm 0.8$ \\
\hline Maximal HR (b min $\left.{ }^{-1}\right)$ & $188 \pm 8.2$ & $188 \pm 5.4$ \\
\hline Average $\mathrm{HR}\left(\mathrm{b} \min ^{-1}\right)$ & $164 \pm 8.8$ & $175 \pm 13.9$ \\
\hline Basal blood lactate $\left(\mathrm{mmol} \mathrm{L}^{-1}\right)$ & $1.4 \pm 0.5$ & $1.7 \pm 0.4$ \\
\hline Maximal blood lactate $\left(\mathrm{mmol} \mathrm{L}^{-1}\right)$ & $13.7 \pm 2$ & $11.9 \pm 0.8$ \\
\hline Recovery blood lactate $\min .2\left(\mathrm{mmol} \mathrm{L}^{-1}\right)$ & $13.4 \pm 1.8$ & $10.5 \pm 1.4$ \\
\hline Recovery blood lactate min. $4\left(\mathrm{mmol} \mathrm{L}^{-1}\right)$ & $12.7 \pm 1.7 *$ & $8.7 \pm 1.3 *$ \\
\hline Recovery blood lactate min. $6\left(\mathrm{mmol} \mathrm{L}^{-1}\right)$ & $11.4 \pm 1.8 *$ & $7.5 \pm 1.6 *$ \\
\hline Recovery blood lactate $\min .8\left(\mathrm{mmol} \mathrm{L}^{-1}\right)$ & $10.4 \pm 1.4 *$ & $6.3 \pm 1.3 *$ \\
\hline Recovery blood lactate min. $10\left(\mathrm{mmol} \mathrm{L}^{-1}\right)$ & $8.9 \pm 1.6 *$ & $5.6 \pm 1.1 *$ \\
\hline
\end{tabular}




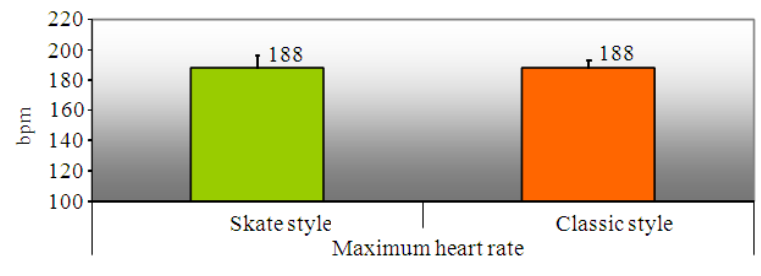

Fig. 4: Comparison of maximum heart rate in skate and classical style

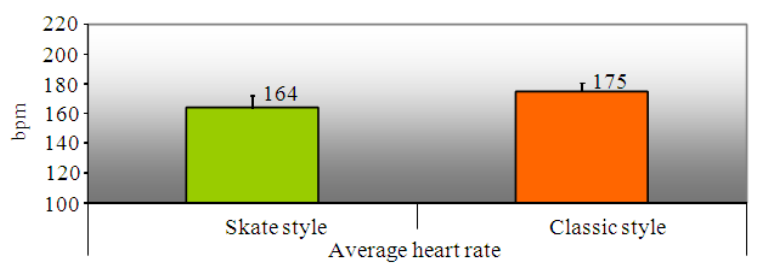

Fig. 5: Comparison of average heart rate in skate and classical style

Figure 4 shows the peak Heart Rate (HR) measured during race. No significant differences were found between classical and skate techniques (skate style: 188 \pm 8.2 vs. classical style: $188 \pm 5.4$ beats per minute, respectively). Figure 5 describes the average HR. The average HR was higher in the classic style $(175 \pm 13.9$ bpm) when compared with the skate style (164 \pm 8.8 bpm), although no significant differences were found between the two styles.

\section{DISCUSSION}

The present study evaluated the performance of elite regional young cross-country skiers comparing the ski skating with the classical style techniques during a national race. Several studies investigated the parameters of different skiing techniques, but most of them were performed in the laboratory under simulated race conditions.

The results recorded in this study revealed differences in the performance between both skiing techniques. Although skate and classical styles attained the same maximal HR, low HR during skate skiing may have been recorded in the downhill zones of the circuit, where only the legs were involved. On the other hand, classical style induces higher frequency movements. Similar investigations highlighted these differences in the average heart rate between the two styles (Hoffman and Clifford, 1990; La Roche et al., 2010).

Differences in blood lactate levels have been recorded, although they were not statistically significant. The free technique results in higher blood lactate concentrations than classical technique under similar race conditions. These values suggest a higher skate skiing muscle activity, while in the classical technique a lower number of muscles were involved. Similar blood lactate levels have been reported by La Roche and colleagues (La Roche et al., 2010) during submaximal roller skiing treadmill protocols.

The lactate concentration calculated immediately after finishing the nordic skiing races is $7-14 \mathrm{~m}^{\prime} \mathrm{Mol} \mathrm{L}^{-1}$ (Welde et al., 2003). In this study, the skate skiing and the classical style lactate concentrations after completing the race were $13.4 \pm 1.9 \mathrm{~m} \cdot \mathrm{MolL}^{-1}$ and $11.98 \pm 0.88 \mathrm{~m} \cdot \mathrm{Mol} \cdot \mathrm{L}^{-1}$ respectively, indicating that the classic technique was more economical than ski skating. Studies on roller skiing performed on a treadmill demonstrate similar results (Hoffman et al., 1990).

Moreover, skate skiing is characterized by a continuous slide producing longer propulsive movements and higher legs strength (Castellar et al., 2006). The classic technique only requires arms to move, while in the skate skiing legs always plays an important role. These differences may be responsible of the higher blood lactate concentration recorded in skate skiing.

At similar lactate concentrations, the skate skiing technique develops a higher speed than the classical style, suggesting the existence of a direct correlation between lactate concentrations and velocity, as other authors pointed out (Vesterinen et al., 2009). In this study, the skate skiing average speed was slightly higher than in the classic style. These values confirm the findings of other investigations where the skate skiing is about 10 and 20-25\% faster than classic style (Stray-Gundersen and Ryschon, 1987; Rusko, 2003). However, in this study differences were lower, probably as a consequence of the age, performance level (kinematic and technical modifications) and training experience to the young skiers who participated in the research. This lack of mechanic efficiency and physical development may induce higher instability, alterations and problems in the dynamic coordination skiing, that may improve the fatigue levels in both techniques (Cignetti et al., 2009a; 2009b).

\section{CONCLUSION}

The results of this study suggest that skate skiing generates a higher impact on the cross-country skier than classical skiing, due to higher blood lactate concentrations during 4, 6, 8 and $10 \mathrm{~min}$ of recovery. 
Although the classical style presents higher resting blood lactate levels, the skate skiing recorded higher blood lactate levels during the race.

The own characteristics of the free technique may be the reason of the lower mean heart rate. These preliminary results suggest the skating action is very fast in junior skiers. From a mechanical and physiological point of view, the skate skiing performance is higher than classical style as a consequence the development a higher speed with a lower energy cost.

\section{ACNOWLEDGEMENT}

The researchers would like to thank the Centre of Winter Sports of Aragon (Spain) for its collaboration and technical assistance provided during the study.

\section{REFERENCES}

Azar, R.S. and Z.M. Shafri, 2009. Mass structure deformation monitoring using low cost differential global positioning system device. Am. J. Applied Sci., $\quad 6$ : 152-156. DOI: 10.3844/ajassp.2009.152.156.

Castellar, C., F., Juvilla and J. A. Casajas, 2006. CD Na1 Congreso Internacional de las Ciencias Deportivas.

Cignetti, F., F. Schema, P.G. Zanone and A. Rouard, 2009a. Dynamics of coordination in cross-country skiing. Hum. Mov. Sci., 28: 204-217. DOI: 10.1016/j.humov.2008.11.002 PMID: 19268379

Cignetti, F., F., Schema and A. Rouard, 2009b. Effects of fatigue on inter-cycle variability in crosscountry skiing. J. Biomech., 42: 1452-1459. DOI: 10.1016/j.jbiomech.2009.04.012 PMID: 19446817
Hoffman, M.D. and P.S. Clifford, 1990. Physiological responses to different cross country skiing techniques on level terrain. Med. Sci. Sports Exerc., 22: 841-848. DOI: 10.1249/00005768199012000-00017 PMID: 2287263

Hoffman, M.D., P. S., Clifford, P. J. Foley and A. G. Brice, 1990. Physiological responses to different roller skiing techniques. Med. Sci. Sports Exerc., 22: 391-396. PMID: 2381308

La Roche, D.P., M. Amann and K.W. Rundell, 2010. Grade influences blood lactate kinetics during cross-country skiing. J. Strength Condit. Res., 24: 120-127. DOI: 10.1519/JSC.0b013e3181c3b429 PMID: 19924008

Rusko, H., 2003. Cross Country Skiing. 1st Edn., Wiley-Blackwell, Mass, Malden, ISBN: 0632055715, pp: 198.

Stray-Gundersen, J. and T.W. Ryschon, 1987. Economy of skating versus classical rollerskiing. Abstract. Med. Sci. Sports Exerc., 19: S46. DOI: 10.1249/00005768-198704001-00275

Vaananen, I. 2004. Physiological responses and mood states after daily repeated prolonged exercise. J. Sports Sci. Med., 3: 1-43.

Vesterinen, V., J. Mikkola, A. Nummela, E. Hynynen and K. Hakkinen, 2009. Fatigue in a simulated cross-country skiing sprint competition. J. Sports Sci., 27: 1069-1077. DOI: 10.1080/02640410903081860 PMID: 19847690

Welde, B., E. Eversten, V. Heimburg and J.I. Medbo, 2003. Energy cost of free technique and classical cross-country skiing at racing speeds. Med. Sci. Sports Exerc., 35: 818-825. PMID: 12750592 